\title{
DIAGNOSTIC DILEMMA OF SARCOIDOSIS: A CASE REPORT MASQUERADING AS TUBERCULOSIS
}

\author{
SHAYMAL SARKAR ${ }^{1}$, MD. DAHARUL ISLAM ${ }^{2}$, MARWA KASHEM MUNA $^{3}$, S M TAJDIT RAHMAN ${ }^{4}$, MD. $^{\circ}$ \\ AZIZUL HOQUE ${ }^{5}$, PARTHAYA GHOSH ${ }^{6}$
}

\begin{abstract}
Sarcoidosis is a chronic multisystem disorder of unknown etiology characterized by formation of granulomata within affected organs and consequent distortion of their normal architecture. Typically, these are non-caseating epithelioid granulomata involving organized collections of activated macrophages and T lymphocytes. In countries where tuberculosis is endemic, sarcoidosis is often misdiagnosed and mistreated as tuberculosis. We present case report of a 47-year-old female who presented with 2 years history of recurrent, multiple nodules with occasional joint pain \& fever and had received anti-tubercular therapy without any improvement. A diagnosis of sarcoidosis is made finally and she was managed accordingly.
\end{abstract}

Received: 10 February 2016

Accepted: 28 December 2016

\section{Introduction}

Sarcoidosis primarily affects individuals between 30 and 50 years of age. Sarcoidosis has a high predilection for the lymph nodes (99\%), lungs (90\%), myocardium (74\%), liver $(60 \%)$, and to a lesser extent the skin $(25 \%)$ and eyes $(25 \%)^{1-5}$. With the exception of the liver, gastrointestinal tract involvement is uncommon. The diagnosis is based on the consistent clinical and radiological findings, supported by histological picture. In approximately one-half of cases, the disease is detected incidentally by radiographic abnormalities noted on a routine chest $x$-ray prior to the development of symptoms. Approximately 20 to $35 \%$ of patients with sarcoidosis have cutaneous involvement. Although cutaneous may present at any time during the course of the disease, they often present early after disease onset. Cutaneous sarcoidosis is known as one of the "great imitators" in dermatology as lesions may assume a vast array of morphologies, masquerading as a wide range of disorders from benign appendageal growths to malignant Kaposisarcoma ${ }^{6-10}$. Therefore, delayed or missed diagnosis often occurs because it may resemble leprosy, tuberculosis, lymphoma cutis [pseudolymphoma] and other granulomatous diseases. We present here a case of systemic sarcoidosis which presented as a diagnostic problem.

\section{Case Report}

A 47 years old house wife presented for the first time in 2014 with multiple subcutaneous nodules in both upper limb for 6 months associated with pain in multiple large \& small joint. She had also experienced occasional low grade fever with no typical evening rise, no chills $\&$ rigor, weight loss. A biopsy from skin nodule revealed fibro-collagenous tissue with inflammatory cells suggestive of rheumatoid nodule (20/09/2014), but there were no other findings that contribute to rheumatoid arthritis. So another biopsy was taken which said caseating granuloma suggestive of tuberculosis (23/09/2014). She was an asthmatic patient with seasonal exacerbation but that time there was no respiratory distress or cough. She was prescribed anti tubercular drug $\&$ she had completed treatment but her symptoms did not improve. In 2016 she again came with generalized weakness, heaviness $\&$ pain in right side of abdomen $\&$ persistence of skin nodules. On general examination there was mild anemia, multiple nontender nodules of different size, largest one measuring $3 \times 2.5 \mathrm{~cm}$, fixed with overlying skin. Her vitals were within normal range. There is no lymphadenopathy \& thyromegaly. Systemic examination revealed nontender hepatomegaly of 5 $\mathrm{cm}$ with regular margin \& smooth surface. The

1. Professor of Medicine, Dhaka Medical College,Dhaka.

2. Assistant Professor, Department of Medicine, Sir Salimullah Medical College, Dhaka.

3. Honorary Medical Officer, Sir Salimullah Medical College Mitford Hospital.

4. MBBS; 27, Navana Garden, Kallyanpur, Dhaka.

5. Associate Professor, Department of Endocrionology, Sirajgonj Medical College, Sirajgonj.

6. Assistant Registrar (Medicine), Sir Salimullah Medical College Mitford Hospital.

Address of Correspondence: Dr. Md. Daharul Islam, Mobile No.: 01718-517833, Email: islamdaharul (a)yahoo.com

Bangladesh J Medicine 2017; $28: 50-52$ 
neurological, cardiovascular and chest examinations were unremarkable.

Blood count and peripheral blood film revealed normocytic normochromic anemia. Biochemical tests showed the $\mathrm{Hb}$ level was $8.0 \mathrm{gm} / \mathrm{dl}$ with ESR $68 \mathrm{~mm}$ in $1^{\text {st }}$ hour. Serum creatinine, glucose, uric acid, albumin and electrolytes were within normal limit. Liver enzymes, viral markers and thyroid function test showed no abnormality. Tuberculin test and sputum smear examination by $\mathrm{ZN}$ staining for AFB was negative. Serum calcium is $9 \mathrm{mg} / \mathrm{dl}$, but angiotensin converting enzyme level was $613 \mathrm{U} / 1$ which is very high. CRP, RA test, ANA was negative.

A chest Xray showed bilateral pulmonary inflammatory lesion. However, a computed tomography (CT) scan of the chest demonstrated predominant peribronchial and perivascular markings involving all segments of both lung with hilar \& pericarinal lymphadenopathy(figure 1).

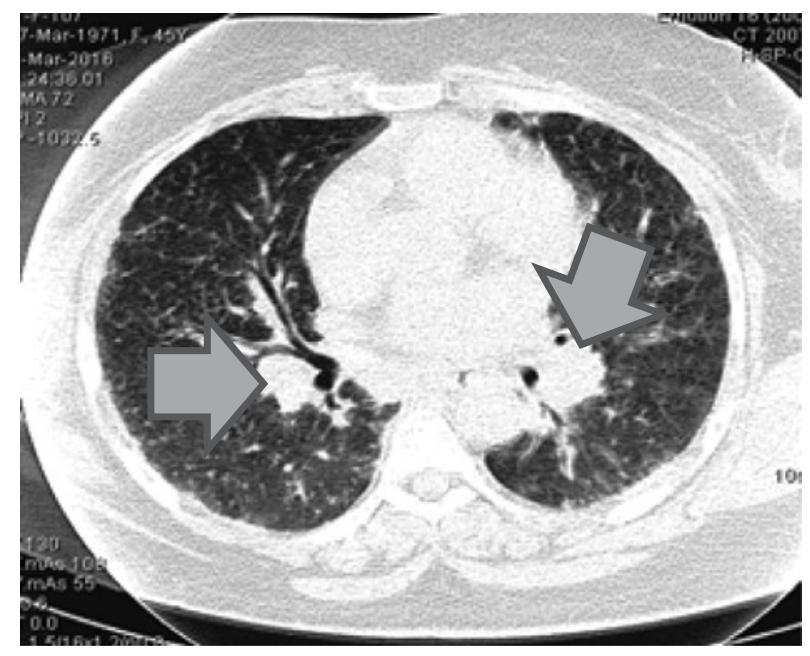

Fig.-1: CT scan of chest showing bilateral hilar lymphadenopathy \& prominent bronchovascular markings.

An ultrasonogram of abdomen was done \& liver biopsy was taken which said noncaseating granuloma made of epitheloid cell \& multinucleated giant cell with scanty lymphocyte \& no AFB was seen in fite faraco stain. Histopathology suggested sarcoidosis (figure $2,3)$.

Biopsy from skin nodule was also taken that revealed granuloma suggestive of sarcoidosis. Pulmonary function test was done which showed: $1.85 \mathrm{~L}(2.5 \mathrm{~L})$; FEV1:1.64L(2.1L); FEV1\%:88.85\%. It clearly indicates restrictive lung disease. Lastly, a diagnosis of sarcoidosis was made and oral steroid was given as treatment.

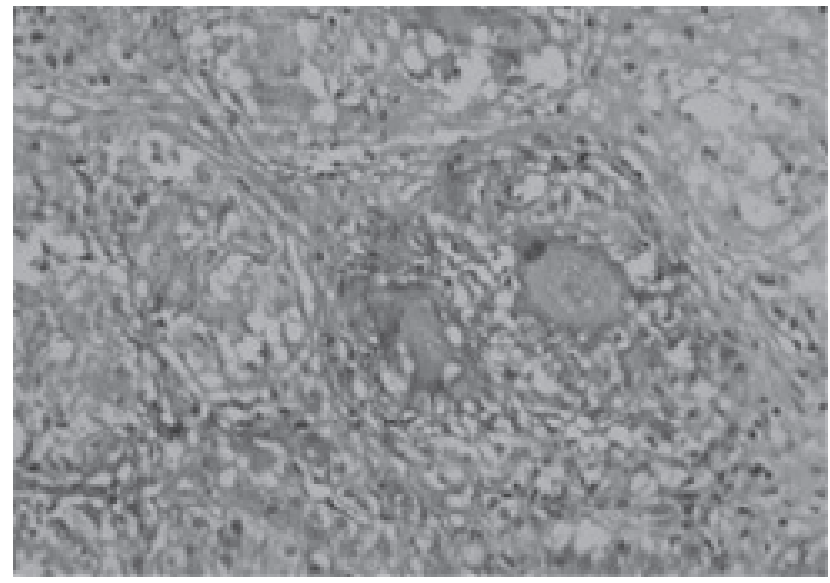

Fig.-2: Histopathology of liver showing granuloma.

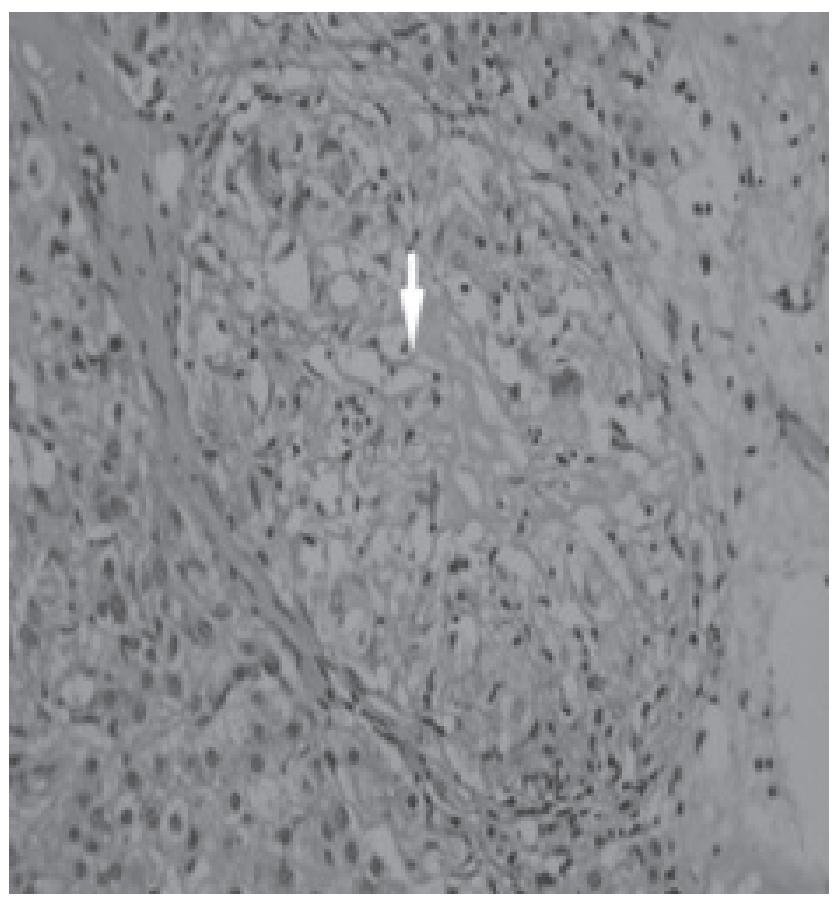

Fig.-3: AFB staining of liver biopsy showing no AFB.

\section{Discussion}

Sarcoidosis however a common disease but often is diagnosed lately. In a country like Bangladesh where tuberculosis is very common, here difficulty arises. This case initially presented as cutaneous sarcoidosis \& later with hepatic involvement. The exact etiology causing sarcoidosis is not clear. Distinction from tuberculosis can be difficult and the two diseases may co-exist on occasion ${ }^{11-12}$.

Cutaneous sarcoidosis may occur at any stage of the disease but most often it is present at the onset of disease ${ }^{6}$. Recognition of cutaneous lesions is important because they provide a visible clue to the 
diagnosis and are an easily accessible source of tissue for histologic examination. However cutaneous lesions may masquerade as a wide range of disorders (leprosy, tuberculosis, lymphoma cutis, lupus vulgaris, leishmania etc) leading to missed and delayed diagnosis as was noted in the present case ${ }^{7-}$ 8 . Patients with cutaneous sarcoidosis are usually asymptomatic. Skin lesions are polymorphous and are classified into three basic groups, erythema nodosum, scar sarcoidosis and skin sarcoid ${ }^{8}$.

Macules, papules, and plaques may arise as single isolated lesions or in crops. Lesions commonly involve the nape of the neck and upper back, extremities, trunk and may appear in scars and tattoos. Lupus pernio, the term for sarcoidosis related indurated, lumpy, violaceous lesions on the nose, cheeks, lips, and ears, can be disfiguring, eroding into underlying cartilage and bone. Lupus pernio is more common in women than in men and is associated with chronic disease and extra pulmonary involvement ${ }^{6}$. Erythema nodosum occurs in about $10 \%$ of patients with sarcoidosis and usually lasts for about 3 weeks. Biopsy specimens of erythema nodosum lesions show nonspecific septal panniculitis, which neither confirms nor negates the diagnosis of sarcoidosis.

Clinical studies indicate involvement of the liver and spleen in approximately $20-30 \%$ of patients affected with sarcoidosis and their detection should be based on a standardized diagnostic procedure ${ }^{13}$. A cholestatic syndrome characterized by pruritus and jaundice, hepatic failure, or portal hypertension can develop; yet liver involvement is usually clinically silent. Detection of hepatic and splenic lesions on computed tomography is described in 5\% and $15 \%$ of patients, respectively. Nearly $60 \%$ of patients with hepatic manifestations of sarcoidosis have constitutional symptoms such as fever, night sweats, anorexia, and weight loss. Portal hypertension with variceal bleeding, a hepatopulmonary syndrome with refractory hypoxemia, and cirrhosis leading to liver failure occur in only $1 \%$ of patients with sarcoidosis. ${ }^{14}$

Most patients with pulmonary sarcoidosis do not require treatment. Asymptomatic CXR infiltrates are usually monitored. Immunosuppressive treatment are therefore prescribed for Increasing symptoms, deteriorating PFTs, and worsening CXR infiltrates, cardiac sarcoidosis, neurosarcoidosis, sightthreatening ocular sarcoidosis, hypercalcaemia, lupus pernio, splenic, hepatic, or renal sarcoidosis. Treatment initially started with steroids, high dose of oral prednisolone $40 \mathrm{mg} /$ day is given for 4 week $\&$ then tapered maintenance dose is $5-20 \mathrm{mg} /$ day.

\section{References:}

1. Crystal RG. Sarcoidosis. In: Fauci AS, Braunwald $\mathrm{E}$, Isselbacher KJ, et al. eds. Harrison's Principles of Internal Medicine. 15th Ed. New York, NY, USA: McGraw-Hill Inc, 2001:1969-1974.

2. Mikami R, Hosoda H, Odaka M. Sarcoidosis. Jpn J Clin Med 1983; 41:1443.

3. Caceres M, Sabbaghian MS, Braud R, Wilks S,Boyle M. Pancreatic sarcoidosis: unusual presentation resembling a periampullary malignancy. Curr Surg 2006; 63:179. [PMID16757369]

4. Siavelis HA, Herrmann ME, Aranha GV, Garcia G, Eubanks T, Reyes CV. Sarcoidosis and the pancreas. Surgery 1999; 125:456-61. [PMID 10216537]

5. Newman LS, Rose CS, Maier LA. Sarcoidosis. N Engl J Med 1997; 336: 1224-34.

6. Mana J, Marcoval J, Graells J, Salazar A, Peyri J, Pujol R. Cutaneous involvement in sarcoidosis: Relationship to systemic disease. Arch Dermatol 1997; 133: 882-8.

7. Samtsov AV. Cutaneous sarcoidosis. Int J Dermatol 1992; 31: 385-91.

8. Katta R. Cutaneous sarcoidosis: A dermatologic masquerader. Am Fam Physician 2002; 65: 1581-4.

9. Ghorpade A, Ramanan C. Cutaneous sarcoidosis. Indian J Dermatol Venereol Leprol 1996; 45: 459-63

10. Thaipisuttikul Y, Kateruttanakul P. Sarcoidosis mimics lepromatous leprosy: A case report. J Med Assoc Thai 2007; 90 [1]: 171-4.

11. Gaitonde SD, Balakrishnan C,Joshi VR. Sarcoidosis: TB or not TB?Ann Rheum Dis. 2003; 62: 495; author reply.

12. Singh M, Kothur K. Pulmonary sarcoidosis masquerading as tuberculosis. Indian Pediatr. 2007; 44: 615-617.

13. skodric'-trifunovic' $v$, vucinic' $v$, colovic' $r$, videnovic' $j$, zugic' $v$, stojsic' J. Liver and splenic sarcoidosis:diagnostic procedures. Med pregl 2004;57: 462466.

14. Harder H, Buchler MW, Frohlich B, et al. Extrapulmonary sarcoidosis of liver and pancreas: a case report and review of literature. World $\mathrm{J}$ Gastroenterol 2007;13: 2504-9. 